\title{
Embodying self-compassion within virtual reality and its effects on patients with depression
}

Caroline J. Falconer, Aitor Rovira, John A. King, Paul Gilbert, Angus Antley, Pasco Fearon, Neil Ralph, Mel Slater and Chris R. Brewin

\section{Background \\ Self-criticism is a ubiquitous feature of psychopathology and can be combatted by increasing levels of self-compassion. However, some patients are resistant to self-compassion.}

\section{Aims}

To investigate whether the effects of self-identification with virtual bodies within immersive virtual reality could be exploited to increase self-compassion in patients with depression.

\section{Method}

We developed an 8-minute scenario in which 15 patients practised delivering compassion in one virtual body and then experienced receiving it from themselves in another virtual body.

\section{Results}

In an open trial, three repetitions of this scenario led to significant reductions in depression severity and self-criticism, as well as to a significant increase in self-compassion, from baseline to 4-week follow-up. Four patients showed clinically significant improvement.

\section{Conclusions}

The results indicate that interventions using immersive virtual reality may have considerable clinical potential and that further development of these methods preparatory to a controlled trial is now warranted.

\section{Declaration of interest}

None.

\section{Copyright and usage}

(c) The Royal College of Psychiatrists 2016. This is an open access article distributed under the terms of the Creative Commons Attribution (CC BY) licence.
Self-criticism is known to be one of the major psychological factors that creates vulnerability and influences recovery and maintenance of depression. ${ }^{1,2}$ Innovative and easily disseminable interventions that address this, and in particular support long-term change for individuals, are urgently needed. Immersive virtual reality can potentially provide this and we report a proof-of-concept study applying one aspect of this technology, virtual embodiment.

When a life-sized virtual body substitutes a person's real body in immersive virtual reality, it typically generates an illusion of body ownership over the virtual body. The illusion of body ownership is the perceptual illusion that the body is one's own and relies on a first-person perspective view of the body $y^{3,4}$ and synchrony between touches seen on the virtual body and felt on the real body ${ }^{5}$ or synchrony between real and virtual body movements through realtime motion capture. ${ }^{6}$ Recent evidence shows that embodiment has a variety of physiological and psychological consequences that indicate that the person has identified with or taken on attributes of the virtual body, including changes in size perception after embodiment in a child body, and changes in implicit racial attitudes after embodiment in a body with a different skin colour. ${ }^{7-10}$

We previously developed a scenario designed to exploit the potential of virtual embodiment to increase feelings of selfcompassion in healthy individuals high in self-criticism. ${ }^{11}$ In the first phase of our immersive virtual reality scenario, participants interacted compassionately with a crying virtual child while embodied in a virtual adult body. In the second phase, one group of participants then embodied the child body and could experience a recording of their compassionate gestures and words being delivered to them from this (child) embodied first-person perspective. By having participants embody an adult and then a child virtual body in succession, our scenario effectively provided a self-to-self situation enabling participants to deliver compassionate sentiments and statements to themselves. Consistent with predictions, this condition resulted in a significantly greater increase in self-compassion than a control condition in which participants saw the same gestures and heard the same words but from a non-embodied, third-person perspective. Self-criticism decreased significantly in both conditions. ${ }^{11}$ The current study uses an uncontrolled case series to assess whether this methodology could also potentially be of benefit in clinical depression.

\section{Method}

\section{Experimental design and participants}

The study utilised a case series with varying baseline length prior to the intervention. Patients were recruited from a local National Health Service Improving Access to Psychological Therapies service. Inclusion criteria were: meeting DSM-IV criteria for current major depressive disorder, according to the Structured Clinical Interview for DSM-IV, ${ }^{12}$ and reporting depressive symptoms at a stable level prior to treatment. Exclusion criteria were: psychotic disorders, organic brain disease, high risk of self-harm or suicide, current substance misuse and lack of fluency in English. Of 18 eligible patients who started treatment, 1 did not complete due to time commitments and 1 did not complete because she found hearing her voice played back to her aversive.

The sample comprised ten women and five men, with an average age of 32 years (range 23-61). All were White except for one Asian male. Four patients had had 1-2 previous episodes of depression, and the remainder reported more episodes with an average of 4 (overall range 1-6). Eight patients reported the onset of their first episodes in their late teens, five in their early 20s, and two in their 30s. Ten patients were currently on antidepressant medication, seven were currently receiving psychological therapy, seven were awaiting therapy and one had finished a course of therapy. One patient had comorbid attention-deficit hyperactivity disorder, one comorbid obsessive-compulsive disorder, and one comorbid obsessive-compulsive disorder and an eating disorder.

\section{Measures}

\section{Severity of depression}

The primary outcome measure was the Patient Health Questionnaire-9 (PHQ-9), ${ }^{13}$ a measure often used to assess outcomes in psychological treatments for depression. ${ }^{14}$ It assesses symptoms 
over the previous 2 weeks and comprises 9 items scored 0 ("not at all'), 1 ('several days'), 2 ('more than half the days') or 3 ('nearly every day'). A reduction of at least 5 points indicated reliable change and this reduction plus a follow-up score of 9 or less indicated clinically significant change. ${ }^{15}$ More transient fluctuations in depressed mood during the baseline and intervention periods were assessed with the Zung Self-Rating Depression Scale (SDS). ${ }^{16}$ The Zung SDS comprises 20 items scored 1 ('a little of the time'), 2 ('some of the time'), 3 ('good part of the time') or 4 ('most of the time') and was modified to be rated with respect to the previous 2 days.

\section{Self-Compassion and Self-Criticism Scale}

The Self-Compassion and Self-Criticism Scale (SCCS) ${ }^{17}$ consists of five scenarios that are potentially self-threatening and can elicit varying degrees of self-criticism or self-compassion (e.g. 'You arrive home to find that you have left your keys at work'). Participants are instructed to imagine that these scenarios are happening to them now and rate on 7-point scales from 1 ('not at all') to 7 ('highly') the extent to which they would react to themselves in a harsh, contemptuous, critical, soothing, reassuring, and compassionate manner. The positive ratings are summed to generate the SelfCompassion Scale (range 15-105) and the negative ratings generate the Self-Criticism Scale (range 15-105). The scale has good internal reliability with Cronbach's alphas of 0.91 and 0.87 respectively, and is sensitive to change. ${ }^{11,17,18}$ Mean scores on self-criticism at baseline were markedly elevated relative to the healthy development sample described by Falconer et al, ${ }^{17} t(426)=3.11, \quad P=0.002$. In contrast, self-compassion scores in the patient sample were comparable with the healthy sample, $t(426)=0.52, P=0.60$.

\section{Fears of Compassion Scales}

Three related scales assess traits consisting of fear of experiencing compassion for oneself ( 15 items: example, 'I fear that if I am more self-compassionate I will become a weak person'), fear of receiving compassion from others (13 items: example, 'When people are kind and compassionate towards me I feel anxious or embarrassed') and fear of experiencing compassion for others (10 items: example, 'People will take advantage of me if they see me as too compassionate'). ${ }^{19}$ All items are rated on a 5 -point scale from 0 ('don't agree at all') to 4 ('completely agree'). High internal reliability (coefficient alpha 0.78-0.85) and validity data have been reported. Comparing this sample's mean scores at baseline with those of the students reported by Gilbert et $a l^{19}$ indicated that patients had greater fear of compassion for self, $t(15.1)=4.25, P<0.001$, and fear of compassion from others, $t(14.7)=4.62, P<0.001$, but less fear of compassion for others, $t(15.1)=-2.65, P<0.05$.

\section{Virtual Reality Experience Questionnaire}

This questionnaire (Appendix) was designed to assess the experience of being in the virtual environment. The question used to assess presence was based on previous virtual reality experiments. ${ }^{20}$ The illusion of body ownership as an adult and as a child were based on two questions (adult session 1 intercorrelation $r(15)=0.69$, $P=0.004$; child session 1 intercorrelation $r(15)=0.78, P=0.001$ ), adapted from questions used in earlier virtual reality studies. ${ }^{8,9}$ Other questions corresponded to aspects of the compassion scenario: recognising the adult as oneself when embodied in the child ( 3 items, session 1 coefficient alpha $=0.66$ ), and the experience of feeling comforted when embodied in the child ( 3 items, session 1 coefficient alpha $=0.68$ ). All items were scored on a scale ranging from -3 ('not at all') to +3 ('very much so'), except for the single item on presence where -3 represented '(I felt like I was in) the lab taking part in an experiment' and 3 represented '(I felt like I was in) a room where there was a child crying'.

\section{Virtual environment}

The virtual room was created using Autodesk $3 \mathrm{~d}$ Max. It was an accurate 3D model of the actual room where the experiment was carried out. The virtual furniture (curtain and door) also matched the placement and size of the actual furniture. The only addition was a virtual mirror where the participants could see a reflection of their virtual body in the virtual environment. The virtual characters used, both adult and child, were acquired from Rocket box Studios. The scenario was implemented with the Unity 3D 4 game engine.

The participant's head was tracked with a 6-DOF InterSense IS-900, a high-rate low-latency tracking system that allows the system to know the participant's head position and orientation and thus adjust the imagery to their perspective in real time. The rest of the body was tracked with Natural Point's Optitrack system with 37 light reflective passive markers attached to the participant's body. Twelve V100 infrared Optitrack cameras captured the tracking volume and body suits from Natural Point were used. To deliver the 3D imagery, we used an nVidiaQuadro 4000 graphics card and annVis Sx111 head-mounted display (HMD). It has two screens built in with a resolution of $1280 \times 1024$ each. They provide a total horizontal field of view of 102 degrees. The HMD is designed in such a way that each eye can only see one of the screens. On each frame of the video, the scene is rendered twice, once on each screen taking into account the disparity between the eyes, providing a stable, immersive percept with stereoscopic depth.

\section{Procedure and materials}

Participants were screened to determine whether they met study inclusion and exclusion criteria. They then attended an initial session to acquaint them with the virtual reality equipment. They were also presented with a video of the researcher participating in the virtual reality task (Supplementary Video S1). ${ }^{11}$ During this session, they completed the Zung SDS, PHQ-9, SCCS and Fears of Compassion Scales. They were then randomly assigned to baseline measurement periods of 4, 5, 6, 8 or 10 days, on each of which they completed the Zung SDS. Prior to commencing the study, the average Zung SDS score was 51.47 (s.d.=7.90) and the average PHQ-9 score was 17.33 (s.d.=3.42), consistent with the presence of major depression. ${ }^{13}$

In the second session, participants were given information concerning compassion and task instructions that presented a staged approach to reducing distress based on current knowledge and practice in compassion-focused therapy. ${ }^{21}$ As in Falconer et al ${ }^{11}$ they were provided with generic sentences that corresponded to each of these three stages (validation, redirection of attention and memory activation) and instructed to memorise them as best they could so as to deliver them slowly, softly and compassionately to the child. Participants practised their lines with the researcher to ensure that they were comfortable expressing themselves. After this, participants were fitted with the HMD and body tracking suit. Embodiment was achieved through a series of simple exercises lasting approximately $2 \mathrm{~min}$ in which they confirmed that the virtual body moved in synchrony with their own movements (visual-motor synchrony) while looking towards themselves directly or in the mirror. Patients were then introduced to the first phase of the scenario, which also lasted approximately $2 \mathrm{~min}$. In this phase, the child was programmed to respond positively to the patient after the delivery of each stage of their compassionate response. The child movements and audio were previously recorded in conjunction with our three-stage compassionate response using a female actor. After their compassionate response, participants were asked to close their eyes to complete the body ownership questions, which were recited to them and their responses were recorded by the researcher. 
In the second phase of the scenario, patients were embodied in the child virtual body and were guided through the same exercises as before to become accustomed to their environment from this new perspective and to the different body. Patients then re-experienced their compassionate response from their new perspective as the child. This included a real-time replay for approximately $2 \mathrm{~min}$ of their original adult body delivering compassion, which portrayed their own body movements and voice. Patients were instructed to merely stand, look and listen to their compassionate response. Participants then exited the virtual reality and completed the virtual reality experience questions including the body ownership questions relating to their time as the child, and the SCCS.

The immersive virtual reality session was repeated a further two times at approximately weekly intervals. After the third session, the primary and secondary outcome measures were assessed. Four weeks after the last session, patients completed the PHQ-9 and SCCS. They were also asked to rate on a 7-point Likert scale from 1 ('not at all') to 7 ('highly') the extent to which they would recommend the study to others, and to comment on their experience of immersive virtual reality.

\section{Ethics statement}

All procedures and materials were approved through the National Research Ethics Service (London-Stanmore section). Written informed consent was obtained from each patient prior to commencement of the study. All patients were given an inconvenience allowance of $£ 36$ for taking part in the study.

\section{Analysis}

All variables were approximately normally distributed except for Zung SDS scores at the end of session 3. Changes in the experience of immersive virtual reality (e.g. degree of body ownership) over the three sessions were tested with a series of repeated-measures ANOVAs. To examine trends over time, the main effect test from the ANOVA was replaced by tests of linear and quadratic effects. Similar analyses were employed with the Zung SDS between start and end of baseline, after each intervention session, and at followup. For consistency, changes in all primary (PHQ-9) and secondary (SCCS, Fear of Compassion Scales) outcome variables were assessed between baseline, the end of the intervention and follow-up using repeated-measures ANOVA. We were unable to obtain end-ofintervention data from two participants. We therefore repeated the analyses on the entire sample, testing for changes between baseline and follow-up only. These yielded identical findings and are not reported further.

\section{Results}

\section{Experience of immersive virtual reality}

Already during session 1 , answers to the presence item (median $=1$; interquartile range $(\mathrm{IQR})=0$ to +2 ) were comparable with previous studies. ${ }^{8}$ Answers at session 1 to the more specific questions on body ownership with respect to the adult body (median $=1.5, \mathrm{IQR}$ $=-0.5$ to +2.0$)$ and body ownership of the child body (median $=1$, $\mathrm{IQR}=-1.5$ to +1.5 ) were comparable with the single session results with self-critical students ${ }^{11}$ exposed to the same scenario. However, at the first session, scores on adult body ownership were significantly higher than corresponding scores on child body ownership, $t(14)=2.17, P<0.05$. This was not the case at subsequent sessions, largest $t(12)=0.76$, not significant.

Table 1 shows changes in the questions relating to presence, body ownership and experience of immersive virtual reality over the three sessions on the various -3 to +3 scales. The main change was a linear increase in recognition of the adult as oneself

\begin{tabular}{|c|c|c|c|}
\hline \multirow[b]{2}{*}{$\begin{array}{l}\text { Experience of virtual } \\
\text { reality scales }\end{array}$} & \multicolumn{3}{|c|}{ Virtual reality session } \\
\hline & Session 1 & Session 2 & Session 3 \\
\hline Presence & $0.77(0.17)$ & $0.23(1.69)$ & $0.69(1.32)$ \\
\hline Adult embodiment & $0.88(1.67)$ & $1.00(1.59)$ & $1.19(1.52)$ \\
\hline Child embodiment & $0.08(1.87)$ & $1.00(1.37)$ & $0.92(1.82)$ \\
\hline Recognise adult as self & $-0.15(1.14)$ & $0.92(1.22)$ & $1.31(0.70)$ \\
\hline Feels comforted as child & $0.82(1.30)$ & $0.82(1.17)$ & $1.28(1.30)$ \\
\hline \multicolumn{4}{|c|}{$\begin{array}{l}\text { a. 'Presence' is the mean of } 1 \text { questionnaire item, 'Adult embodiment' and 'Child } \\
\text { embodiment' are the mean of } 2 \text { questionnaire items, 'Recognise adult as self' is the } \\
\text { mean of } 3 \text { questionnaire items, 'Feels comforted as child' is the mean of } 3 \\
\text { questionnaire items. }\end{array}$} \\
\hline
\end{tabular}

when embodied in the child, $F(1,12)=14.48, P=0.003, \eta_{\mathrm{p}}{ }^{2}=0.55$. There were also linear increases in body ownership as the child, $F(1,12)=3.58, P=0.08, \eta_{\mathrm{p}}{ }^{2}=0.23$, and in the experiences of being comforted while in the body of the child, $F(1,12)=3.60$, $P=0.08, \quad \eta_{\mathrm{p}}{ }^{2}=0.23$, but these were weaker and non-significant. There were no other linear or quadratic effects of interest, largest $F(1,14)=2.18$, not significant.

Scores on adult and child body ownership were strongly positively correlated across all three sessions, smallest $r(15)=0.73$, $P=0.002$. Ratings of feeling comforted while in the body of the child were most consistently correlated with recognising the adult as oneself, although the correlation was not significant for session 3 (session 1: $r(15)=0.58, P=0.02$; session 2: $r(14)=0.60, P=0.02$; session 3: $r(13)=0.35, P=0.25)$. On average patients said they would recommend the study strongly to others (mean $=5.5$, s.d.=1.35).

\section{Changes in severity of depression, self-criticism and self-compassion}

Transient changes in depressed mood over the previous 2 days as measured with the Zung SDS were assessed prior to the study $(M=54.80$, s.d. $=4.80)$ and again after the individual baseline period, after each immersive virtual reality intervention session, and at 4week follow-up. A repeated-measures ANOVA found no evidence for linear, quadratic or cubic changes in transient mood over these six measurement points, largest $F(1,12)=2.27, P=0.16$.

The primary outcome measure was the PHQ-9. Scores at baseline, after the immersive virtual reality sessions, and at 4-week follow-up are summarised in Table 2. There was a linear effect of time, $F(1,12)=14.04, P=0.003, \eta_{\mathrm{p}}{ }^{2}=0.54$, such that participants became less severely depressed post-intervention but no significant quadratic effect, $F(1,12)=0.06$, not significant. The withinparticipant effect size from baseline to follow-up controlling for the dependence between means was 1.11 (Cohen's $d z$ ). Of the 15 patients, 5 showed reliable improvement and a further 4 showed clinically significant change.

For self-compassion as measured by the SCCS, there was a significant linear increase in scores, $F(1,12)=6.65, P=0.02$, $\eta_{\mathrm{p}}{ }^{2}=0.36$, and no quadratic effect, $F(1,12)=0.69$, not significant. Scores at follow-up were significantly greater than those of the development sample reported by Falconer et al, ${ }^{17} t(426)=3.83$, $P<0.001$. Likewise, for self-criticism, there was a significant linear decrease in scores, $F(1,12)=23.41, P<0.001, \eta_{\mathrm{p}}{ }^{2}=0.66$, and no quadratic effect, $F(1,12)=0.06$, not significant. Scores at follow-up were significantly lower than those of the development sample reported by Falconer et al, ${ }^{17} t(426)=3.85, P<0.001$.

All the three scales measuring the trait 'fear of compassion' showed a linear decrease over the course of the intervention but not to a significant extent. The largest changes were in 'fear of compassion 


\begin{tabular}{|c|c|c|c|}
\hline \multirow[b]{2}{*}{ Measurements } & \multicolumn{3}{|c|}{ Study time points } \\
\hline & Baseline & Post-virtual reality intervention ${ }^{a}$ & 4-week follow-up \\
\hline Patient Health Questionnaire-9 & $17.54(3.43)$ & $14.23(6.03)$ & $11.69(6.18)$ \\
\hline SCCS: self-criticism & $67.23(23.82)$ & $49.54(20.44)$ & $34.38(16.85)$ \\
\hline SCCS: self-compassion & 35.54 (19.03) & $38.92(19.28)$ & $52.00(16.29)$ \\
\hline Fear of compassion for self & $29.15(11.57)$ & $26.38(8.56)$ & $21.23(10.51)$ \\
\hline Fear of compassion for others & $15.23(8.53)$ & $15.77(9.58)$ & $13.77(9.71)$ \\
\hline Fear of compassion from others & $27.69(9.77)$ & $25.54(8.03)$ & $21.23(7.95)$ \\
\hline
\end{tabular}

for self', $F(1,12)=2.76, P=0.12, \eta_{\mathrm{p}}{ }^{2}=0.19$, and in 'fear of compassion from others', $F(1,12)=3.34, P=0.09, \eta_{\mathrm{p}}{ }^{2}=0.22$. No other linear or quadratic effects were evident, largest $F(1,12)=0.27$, not significant. At follow-up, patients' scores on 'fear of compassion for self' no longer differed significantly from the student sample of Gilbert et al $^{19}$ (95\% CI -0.38 to 10.60$)$.

\section{Discussion}

The most important finding was that there was evidence of significant reductions in depression severity and self-criticism, as well as increases in self-compassion. Second, patients' initial perception of the immersive virtual reality self-compassion scenario was very similar to that of healthy volunteers we studied previously, and their overall experience of it was positive. Third, repeating sessions appeared to deepen some aspects of patients' experience of the scenario. Taken together, the data support the idea that immersive virtual reality is an intervention this clinical group can potentially benefit from.

Why might an intervention aimed at increasing self-compassion be effective in depression? Elevated self-criticism blocks positive affects, especially those associated with self-compassion. ${ }^{22}$ Partly because self-compassion is linked to prosocial caring-based emotions, it is a natural regulator of mood, feelings of self-worth and social connectedness. ${ }^{21}$ Given this increasing research on the role of positive and socially affiliative emotion in the regulation of mental health problems, a number of therapies aimed at cultivating self-compassion have been developed. ${ }^{23-26}$ Directly encouraging self-compassionate feelings can however be difficult for some patients who may feel they do not deserve compassion or find it stimulates aversive memories. ${ }^{19}$ Experiences mediated by immersive virtual reality may help to bypass such reactions.

Compassion is a complex process of empathy, distress tolerance, sympathy, motivation, courage, wisdom and acts of kindness in both inter- and intrapersonal spheres. People with depression with critical internal monologues would not be used to hearing selfdirected compassionate statements in their own voice. We think that our paradigm furnished individuals with knowledge about the nature and purpose of compassion as well as provided them with a phenomenological experience of giving and receiving it. Of particular importance for compassion is motivation. ${ }^{27}$ Our participants, by volunteering to take part in the study, were obviously motivated to learn about it, and we can only assume that this motivation, accompanied by the experience we provided, allowed them to act compassionately towards themselves.

Our results leave a number of important questions about the key elements of the approach unanswered, however: is it the giving of compassion to a vulnerable other, the receiving of compassion, the experience of receiving compassion that appears to come from the self or the more specific experience of receiving this compassion from a first-person perspective? Our previous data ${ }^{11}$ implicated this last possibility, but the absence of a third-person perspective control condition means that this remains to be established in a patient sample.

Although Zung SDS scores did not indicate any change, the PHQ-9 demonstrated a significant linear decrease over the period from baseline through to follow-up with over half of the patients reporting reliable levels of improvement. The amount of change from baseline to the end of the intervention was similar to the amount of change from the end of the intervention to follow-up, suggesting that immersive virtual reality sessions had an indirect effect on self-perceptions that took time to fully develop. Similar results have been reported for other interventions for depression, such as imagery rescripting. ${ }^{28}$

Even though in large samples the Zung SDS and PHQ-9 tend to be positively correlated, results for individual patients often show large discrepancies. ${ }^{29}$ One reason for the discrepancy in this study is that whereas the Zung SDS ratings focused on the past 2 days, the PHQ-9 required patients to take a broader perspective and assess their mood over a 2-week period, as required for a diagnosis of clinical depression. The response options of the PHQ-9 were also worded more precisely than those of the Zung SDS, for example, requiring patients to consider whether symptoms were present on more days than not. A plausible interpretation of the discrepancy is that transient feelings of depression as measured by the Zung SDS were stable across the whole intervention, that is, patients continued to experience equally strong negative mood some of the time, but the duration of these negative moods, as measured by thePHQ-9, was shorter. Previous research comparing the PHQ-9 with another commonly used outcome measure (the Beck Depression Inventory II) suggested that both had equivalent sensitivity to change in the context of a clinical trial for depression. ${ }^{30}$ The amount of change patients reported compares favourably with the results of other very brief interventions. ${ }^{31}$

Even if the PHQ-9 results are given greater weight than those involving the Zung SDS, without a control group it cannot be concluded that the intervention is responsible for the clinical improvement. Nevertheless, this possibility is strengthened by the brevity of the intervention relative to patients' history of depression and by the concurrent improvement in the targeted process variables, namely the increase in patients' self-compassion and reduction in self-criticism in response to hypothetical situations. It is important to note that these changes did not just result in patients scoring equivalently to the healthy samples used in the development of the SCCS but resulted in substantially higher levels of selfcompassion and substantially less self-criticism than this healthy group.

This impression was supported anecdotally by patients' comments about their experience of treatment. Feedback after completion of the virtual reality sessions and at 1-month follow-up clearly showed a consensus around an 'enjoyable', 'interesting' and 'helpful' experience. Some patients explicitly reported the positive 
benefits of the immersive virtual reality sessions on their mood and arousal, either immediately after the sessions or at follow-up. For example, one patient explicitly stated that the sessions gave her an awareness and acceptance that "we are all human and vulnerable and it's ok to be vulnerable as an adult'. This statement demonstrates the patient's understanding of compassion, which emphasises suffering as part of the human condition, and the acknowledgement and self-acceptance of one's own experience within this framework.

A recurrent theme in patients' feedback concerned the positive experience that immersive virtual reality offered in relation to 'the self'. Patients commented that it allows you to 'step out of yourself, apply yourself and "bear witness" to yourself'. These comments are consistent with our intention of creating a scenario to alter the relationship between different aspects of the self, and to counter negative self-attitudes. Similar observations together with the finding that expressions of emotion in human-like avatars are perceived similarly to human emotions ${ }^{32}$ are driving a variety of novel assessments and treatments for clinical conditions as diverse as auditory hallucinations, ${ }^{33}$ paranoia $^{34}$ and pain. ${ }^{10,35}$ These techniques also hold considerable promise for strengthening positive emotions, for example, most recently in the realisation of self-self dialogue where the participant is afforded self-counselling via alternately embodying different bodies. ${ }^{36}$

Key to all psychological therapy is the ability to generalise knowledge to real-world situations. Twelve of the patients explicitly reported thinking about the scenario outside the immersive virtual reality sessions. Of these, some reported merely reflecting on the experience, discussing it with people and how it had or might help them in the future. One person mentioned that she had discussed the study with 'a couple of dozen people'. Many patients reported that they recalled their immersive virtual reality experience when trying to comfort others. Most importantly, however, numerous patients reported thinking about their experience and applying it to themselves while upset. For example, one patient reported that her participation in the immersive virtual reality helped her to be more self-compassionate when reflecting on herself in her cognitivebehavioural therapy sessions, a process that she had struggled with and found particularly distressing prior to participating.

Depression is a condition characterised by extremely high levels of self-focused attention. ${ }^{37}$ It is therefore reasonable to wonder whether the capacity to respond to virtual environments might be correspondingly reduced, but ratings of presence, or susceptibility to the body ownership illusion, were similar to those reported in Falconer et al. ${ }^{11}$ No changes were observed in presence generally, or in the sense of identification with the adult virtual body, over the repeated sessions. However, embodiment in the child body was initially lower than in the adult one, subsequently equalising. Both these findings suggest that patients took some time to get used to the idea of embodiment, and particularly in relation to the child virtual body. Consistent with this, there was an increase in reported ability while embodied in the child to recognise the adult body as oneself. We may tentatively conclude that patients do require a degree of practice with these complex scenarios in order to understand and derive full benefit from them.

The results need to be understood within the context of the study limitations. Chief among these was the relatively small number of patients, the absence of the kind of control condition we previously employed, ${ }^{11}$ the repetition of a single immersive virtual reality scenario and the lack of prior information about what factors would make the experience more or less effective. Other limitations include the repeated use of the SCCS, which may have diminished the validity of the measure, and the possible influence of demand characteristics. One of the most striking differences with our previous study using a healthy sample ${ }^{11}$ was that every patient reported that it was 'horrible', 'strange' or 'cringing' to hear their own voice, although some patients explicitly stated that this 'gets easier'. Our positive results were obtained despite these experiences but, nevertheless, future studies should consider preparing patients better for this or experiment using alternative voices that may be experienced as more acceptable. Numerous patients also reported a preference for varying the content of the scenarios and improving the aesthetic quality of the virtual reality experience, for example, by better motion tracking and fluidity of movement, and more natural environments and facial features. They also suggested it might be beneficial to make material available to practice with outside the immersive virtual reality sessions and to reflect on the experience more in terms of how it made them feel, what they should be experiencing and how they might improve their next experience.

In summary, this study adds to a growing literature, $, 10,34,35$ suggesting that the exploitation of new knowledge about the effects of embodiment is likely to be of value to patients with several physical and psychological disorders. Although our experimental setup was expensive, equipment that will mimic it effectively is already becoming widely available to consumers at low cost. Future developments in immersive virtual reality offer an alternative that could reduce or eliminate face-to-face therapist time. Given the importance of social resources in determining the outcome of depression, ${ }^{38-40}$ they may also offer an alternative for those experiencing social isolation typical of this condition.

\section{Caroline J. Falconer, PhD, Clinical Educational \& Health Psychology, Aitor Rovira, MSC, Department of Computer Science, John A. King, PhD, Clinical Educational \& Health Psychology, University College London, London, UK; Paul Gilbert, PhD, Mental Health Research Unit, University of Derby, Derby, UK; Angus Antley, PhD, Department of Computer Science; Pasco Fearon, PhD, Clinical Educational \& Health Psychology, University College London, London, UK; Neil Ralph, DClinPsy, Clinical Educational \& Health Psychology, University College London, London, and iCope Camden and Islington Psychological Therapies Service, London, UK; Mel Slater, DSC, Institució Catalana de Recerca i Estudis Avançats, University of Barcelona, Barcelona, Spain, and Department of Computer Science, University College London, London, UK; Chris R. Brewin, PhD, Clinical Educational \& Health Psychology, University College London, London, UK}

Correspondence: Chris R. Brewin, Clinical, Educational, and Health Psychology, University College London, Gower Street, London WC1E 6BT, UK. Email: c.brewin@ucl. ac.uk

First received 13 Sep 2015, final revision 4 Dec 2015, accepted 21 Dec 2015

\section{Funding}

The research was funded by a grant from the UK Medical Research Council (MR/J009210/1).

\section{References}

1 Kannan D, Levitt HM. A review of client self-criticism in psychotherapy. J Psychother Integr 2013; 23: 166-78.

2 Blatt SJ, Zuroff DC. Interpersonal relatedness and self-definition - 2 protypes for depression. Clin Psychol Rev 1992; 12: 527-62.

3 Petkova $\mathrm{Vl}$, Khoshnevis $\mathrm{M}$, Ehrsson $\mathrm{HH}$. The perspective matters! Multisensory integration in egocentric reference frames determines full-body ownership. Front Psychol 2011; 2: 35

4 Slater M, Spanlang B, Sanchez-Vives MV, Blanke O. First person experience of body transfer in virtual reality. PLOS One 2010; 5: e10564.

5 Petkova VI, Ehrsson HH. If I were you: perceptual illusion of body swapping. PLOS One 2008; 3: e3832.

6 Banakou D, Slater M. Body ownership causes illusory self-attribution of speaking and influences subsequent real speaking. Proc Natl Acad Sci U S A 2014; 111: 17678-83.

7 Maister L, Slater M, Sanchez-Vives MV, Tsakiris M. Changing bodies changes minds: owning another body affects social cognition. Trends Cogn Sci 2015; 19: 6-12. 
8 Banakou D, Groten R, Slater M. Illusory ownership of a virtual child body causes overestimation of object sizes and implicit attitude changes. Proc Natl Acad SCi U S A 2013; 110: 12846-51.

9 Peck TC, Seinfeld S, Aglioti SM, Slater M. Putting yourself in the skin of a black avatar reduces implicit racial bias. Conscious Cogn 2013; 22: 779-87.

10 Martini M, Perez-Marcos D, Sanchez-Vives MV. Modulation of pain threshold by virtual body ownership. Eur J Pain 2014; 18: 1040-8.

11 Falconer CJ, Slater M, Rovira A, King JA, Gilbert P, Antley A, et al. Embodying compassion: a virtual reality paradigm for overcoming excessive self-criticism. PLoS One 2014; 9: e111933.

12 First MB, Spitzer RL, Gibbon M, Williams JBW. Structured Clinical Interview for DSM-IV Axis I Disorders-Patient Edition (SCID-I/P, Version 2.0). Biometrics Research Department of the New York State Psychiatric Institute, 1995

13 Kroenke K, Spitzer RL, Williams JBW. The PHQ-9 - validity of a brief depression severity measure. J Gen Intern Med 2001; 16: 606-13.

14 Williams $A D$, Andrews $G$. The effectiveness of internet cognitive behavioural therapy (iCBT) for depression in primary care: a quality assurance study. PLOS One 2013; 8: e57447.

15 McMillan D, Gilbody S, Richards D. Defining successful treatment outcome in depression using the PHQ-9: a comparison of methods. J Affect Disord 2010; 127 $122-9$.

16 Zung WWK. A self-rating depression scale. Arch Gen Psychiatry 1965; 12: 63-70.

17 Falconer CJ, King JA, Brewin CR. Demonstrating mood repair with a situationbased measure of self-compassion and self-criticism. Psychol Psychother 2015; 88: $351-65$.

18 Kamboj SK, Kilford EJ, Minchin S, Moss A, Lawn W, Das RK, et al. Recreational 3,4 methylenedioxy-N-methylamphetamine (MDMA) or 'ecstasy' and self-focused compassion: preliminary steps in the development of a therapeutic psychopharmacology of contemplative practices. J Psychopharmacol 2015; 29: 961-70.

19 Gilbert $\mathrm{P}$, McEwan $\mathrm{K}$, Matos M, Rivis A. Fears of compassion: development of three self-report measures. Psychol Psychother 2011; 84: 239-55.

20 Sanchez-Vives MV, Slater M. From presence to consciousness through virtual reality. Nat Rev Neurosci 2005; 6: 332-9.

21 Gilbert P. Compassion Focused Therapy: Distinctive Features. Routledge 2010.

22 Gilbert P, McEwan K, Gibbons L, Chotai S, Duarte J, Matos M. Fears of compassion and happiness in relation to alexithymia, mindfulness and self-criticism. Psycho Psychother 2013; 84: 239-55.

23 Gilbert $\mathrm{P}$, Irons $\mathrm{C}$. A pilot exploration of the use of compassionate images in a group of self-critical people. Memory 2004; 12: 507-16.

24 Hofmann SG, Grossman P, Hinton DE. Loving-kindness and compassion meditation: potential for psychological interventions. Clin Psychol Rev 2011; 31: 1126-32.

25 Leary MR, Tate EB, Adams CE, Allen AB, Hancock J. Self-compassion and reactions to unpleasant self-relevant events: the implications of treating oneself kindly. J Pers Soc PSychol 2007; 92: 887-904.
26 Neff KD, Germer CK. A pilot study and randomized controlled trial of the mindful self-compassion program. J Clin Psychol 2013; 69: 28-44.

27 Gilbert P. The evolution and social dynamics of compassion. Soc Personal Psychol Compass 2015; 9: 239-54.

28 Brewin CR, Wheatley I, Patel T, Fearon P, Hackmann A, Wells A, et al. Imagery rescripting as a brief stand-alone treatment for depressed patients with intrusive memories. Behav Res Ther 2009; 47: 569-76.

29 Hawley CJ, Gale TM, Smith PSJ, Jain S, Farag A, Kondan R, et al. Equations for converting scores between depression scales (MADRS, SRS, PHQ-9 and BDI-II): good statistical, but weak idiographic, validity. Hum Psychopharmacol 2013; 28 544-51.

30 Titov N, Dear BF, McMillan D, Anderson T, Zou J, Sunderland M.Psychometric comparison of the PHQ-9 and BDI-II for measuring response during treatment of depression. Cogn Behav Ther 2011; 40: 126-36.

31 Williams $A D$, Blackwell SE, Mackenzie A, Holmes EA, Andrews G.Combining imagination and reason in the treatment of depression: a randomized controlled trial of internet-based cognitive-bias modification and internet-CBT for depression. J Consult Clin Psychol 2013; 81: 793-9.

32 de Borst AW, de Gelder B. Is it the real deal? Perception of virtual characters versus humans: an affective cognitive neuroscience perspective. Front Psychol 2015; 6: 576

33 Leff J, Williams G, Huckvale M, Arbuthnot M, Leff AP. Avatar therapy for persecutory auditory hallucinations: what is it and how does it work? Psychosis 2014; 6: 166-76.

34 Freeman D, Antley A, Ehlers A, Dunn G, Thompson C, Vorontsova $\mathrm{N}$, et al. The use of immersive virtual reality (VR) to predict the occurrence 6 months later of paranoid thinking and posttraumatic stress symptoms assessed by self-report and interviewer methods: a study of individuals who have been physically assaulted. Psychol Assess 2014; 26: 841-7.

35 Hoffman HG, Patterson DR, Carrougher GJ. Use of virtual reality for adjunctive treatment of adult burn pain during physical therapy: a controlled study. Clin J Pain 2000; 16: 244-50.

36 Osimo SA, Pizarro R, Spanlang B, Slater M. Conversations between self and self as Sigmund Freud - a virtual body ownership paradigm for self counselling. Sci Rep 2015; 5: 13899

37 Pyszczynski T, Greenberg J. Self-regulatory perseveration and the depressive selffocusing style - a self-awareness theory of reactive depression. Psychol Bull 1987; 102: $122-38$

38 Billings AG, Moos RH. Life stressors and social resources affect posttreatment outcomes among depressed patients. J Abnorm Psychol 1985; 94: 140-53.

39 George LK, Blazer DG, Hughes DC, Fowler N. Social support and the outcome of major depression. Br J Psychiatry 1989; 154: 478-85.

40 Hirschfeld RMA, Cross CK. Epidemiology of depression: psychosocial risk factors. Arch Gen Psychiatry 1983; 39: 35-46. 


\section{Appendix}

\section{Items measuring the experience of immersive} virtual reality

\section{Presence}

I felt like I was in a room where a child was crying as opposed to in a lab taking part in an experiment.

Adult body ownership and agency

I felt as if the body I saw when I looked down was my own body. I felt as if the body I saw when I looked in the mirror was my own body.

Child body ownership and agency

I felt as if the body I saw when I looked down was my own body.

I felt as if the body I saw when I looked in the mirror was my own body.
Child recognises adult as self

I felt like I was in the child's role.

I could recognise myself as the adult when I was the child.

I could recognise myself in the movement of the adult when I was the child.

Child sense of being comforted

I felt like I was giving myself compassion when I was the child.

I felt comforted by myself as the adult when I was the child

I felt reassured by myself as the adult when I was the child. 